\title{
Lipoproteína "a": elevaciones extremas y polimorfismos genéticos, asociación con lesiones cardíacas y vasculares evaluadas por tomografía
}

\author{
Lipoprotein a: Extreme Elevation and Genetic Polymorphism Association with Cardiac and \\ Vascular Lesions Evaluated by Computed Tomography
}

PABLO CORRAL ${ }^{1}$, MARCELO RODRÍGUEZ², SILVINA QUINTANA ${ }^{3}$ DIEGO QUIRÓS4, AUGUSTO SIGISMONDI ${ }^{5}$

\begin{abstract}
RESUMEN
Introducción: La lipoproteína a [Lp(a)] es una partícula compleja, similar a una lipoproteína de baja densidad, asociada con una molécula de apolipoproteína "a" [apo(a)]. La concentración elevada de Lp(a) plasmática se asocia con riesgo aumentado de enfermedad coronaria y de calcificación valvular aórtica. El riesgo inherente a esta relación está determinado principalmente por factores genéticos.

Objetivos: Analizar un grupo de pacientes con elevaciones extremas de Lp(a) [> $100 \mathrm{mg} / \mathrm{dl}$ ], su asociación con calcificaciones cardiovasculares torácicas (valvulares, coronarias, aorta torácica) detectadas mediante tomografía axial computarizada y evaluar tres polimorfismos genéticos vinculados con los niveles de Lp(a) y lesiones en estas tres regiones.

Material y métodos: Se estudiaron 40 pacientes en los que se analizaron los polimorfismos rs 10455872 y rs2048327 mediante high resolution melting y el número de repeticiones de la secuencia KIV del exón 2 del gen LPA mediante qPCR.

El promedio de edad de los pacientes fue de 52,9 años (37\% mujeres) y el valor promedio de Lp(a) fue de $170,4 \mathrm{mg} / \mathrm{dl}$.

Resultados: El 75\% (30/40) de los pacientes presentó al menos una calcificación en la tomografía (valvular, coronaria y/o aorta torácica); de los pacientes con calcificaciones, el $90 \%$ presentaron al menos uno de los factores genéticos asociados con mayor patogenicidad de la $\operatorname{Lp}(\mathrm{a})$.

Conclusión: En un grupo de pacientes con niveles elevados de Lp(a) encontramos un número importante de calcificaciones cardiovasculares torácicas y determinantes genéticos asociados con diferentes isoformas de Lp(a), que podrían ocasionar los niveles elevados de $\mathrm{Lp}$ (a) y el riesgo de desarrollo de lesiones valvulares y/o vasculares.
\end{abstract}

Palabras clave: Lipoproteína - Hiperlipidemia - Polimorfismo genético - Calcificación vascular

\begin{abstract}
Background: Lipoprotein a [Lp(a)] has a complex structure, similar to low density lipoprotein, associated with one molecule of apolipoprotein a [apo(a)]. Elevated plasma levels of $\mathrm{Lp}(\mathrm{a})$ are related to greater risk of coronary artery disease and calcification of the aortic valve. This risk is mainly determined by genetic factors.

Objectives: The aim of this study was to analyze a group of patients with extreme Lp(a) levels [ $>100 \mathrm{mg} / \mathrm{dL}]$ and their association with calcifications of the aortic valve, coronary arteries and thoracic aorta detected by computed tomography scan, and to evaluate three genetic polymorphisms associated with $\mathrm{Lp}$ (a) levels and lesions in these three regions.

Methods: rs10455872 and rs2048327 polymorphisms were analyzed in 40 patients using high resolution melting and the number of KIV-2 repeats in the LPA gene was evaluated using quantitative PCR.

Patient mean age was 52.9 years (37\% women) and mean $\mathrm{Lp}(\mathrm{a})$ was $170.4 \mathrm{mg} / \mathrm{dL}$.

Results: Seventy-five percent of patients (30/40) presented at least one calcification in the computed tomography scan (valves, coronary arteries and/or thoracic aorta), and among them, $90 \%$ had at least one of the genetic factors associated with Lp(a) pathogenicity.

Conclusion: In a group of patients with elevated $\mathrm{Lp}$ (a) levels, we found a significant number of cardiovascular thoracic calcifications and genetic determinants associated with different $\mathrm{Lp}$ (a) isoforms that could be related with elevated Lp(a) levels and high risk of developing valve or vascular lesions.
\end{abstract}

Key words: Lipoprotein - Hyperlipidemia - Polymorphism, Genetic - Vascular Calcification

Rev Argent CARdiol 2017;85:28-33. http://dx.doi.org/10.7775/rac.es.v85.i1.10295

VÉASE CONTENIDO RELACIONADO: Rev Argent Cardiol 2017;85:1-3. http://dx.doi.org/10.7775/rac.es.v85.i1.10232

Recibido: 02/12/2016 - Aceptado: 17/01/2017

Dirección para separatas: Pablo Corral - Rawson 1006 - Mar del Plata, Pcia. de Buenos Aires, Argentina - e-mail:drpablocorral@gmail.com Tel. 00542234974817

Este trabajo ha sido ganador del Premio Fundación Dr. Pedro Cossio en el 42 Congreso Argentino de Cardiología 2016

${ }^{1}$ Farmacología, Facultad de Medicina, Universidad FASTA, Mar del Plata

${ }^{2}$ Clínica Colón, Servicio de Cardiología Clínica, Mar del Plata

${ }^{3}$ Laboratorio de Biología Molecular, Fares Taie Instituto de Análisis, Mar del Plata. Consejo Nacional de Investigaciones Científicas y Técnicas (CONICET)

${ }^{4}$ Clínica Colón, Servicio de Diagnóstico por Imágenes, Mar del Plata

${ }^{5}$ Facultad de Medicina, Universidad FASTA, Mar del Plata 


\begin{tabular}{ll|ll}
\multicolumn{2}{l|}{ Abreviaturas } & \\
ADN & Ácido desoxirribonucleico & ECV & Enfermedad cardiovascular \\
C-HDL & Colesterol transportado por lipoproteínas de alta & HRM & High resolution melting \\
& densidad & LDL & Lipoproteína de baja densidad \\
C-LDL & Colesterol transportado por lipoproteínas de baja & Lp(a) & Lipoproteína a \\
& densidad & PCR & Reacción en cadena de la polimerasa \\
C-noHDL & Colesterol no de lipoproteínas de alta densidad & SNP & Polimorfismo de nucleótido único \\
CT & Colesterol total & TG & Triglicéridos
\end{tabular}

\section{INTRODUCCIÓN}

La lipoproteína “a” $[\operatorname{Lp}(a)]$ consiste en una fracción lipoproteica similar a la lipoproteína de baja densidad (LDL), unida a una glicoproteína, denominada apolipoproteína (a). (1) Niveles elevados (> 30-50 mg/dl) de $\mathrm{Lp}$ (a) se consideran un factor de riesgo causal e independiente para la enfermedad cardiovascular (ECV). (2) Distintas líneas de evidencia han demostrado que la estructura dual de la Lp(a) y sus niveles plasmáticos elevados se asocian con la calcificación de la raíz aórtica, como también con lesiones vasculares ateroscleróticas, sobre todo a nivel del lecho coronario. (3)

El nivel de $\mathrm{Lp}$ (a) puede variar hasta mil veces entre individuos y está determinado principalmente por la variación en el gen que codifica la LPA [apolipoproteína(a)]; (4) de hecho, se considera que la Lp(a) es la lipoproteína con el mayor control genético. (5) Se considera que los individuos con niveles de Lp(a) mayores de $100 \mathrm{mg} / \mathrm{dl}$ padecen de elevaciones extremas de esta lipoproteína y un riesgo asociado mayor. (6)

Existen diferentes isoformas de Lp(a), siendo las pequeñas las específicamente perjudiciales. (4) El factor más influyente en los niveles de Lp(a) y la determinación de sus isoformas es el Kringle denominado IV tipo 2 (KIV-2) en el gen LPA; la repetición de este polimorfismo determina el tamaño de la apolipoproteína (a), y el número de repeticiones se correlaciona inversamente con los niveles plasmáticos de Lp(a). (5)

Adicionalmente, se ha demostrado que el polimorfismo de nucleótido único (SNP) rs10455872 en el gen LPA se asocia directamente con niveles altos de Lp(a) y con un riesgo mayor de calcificación/estenosis de la válvula aórtica en diferentes poblaciones. $(3-5,7)$ Asimismo, a principios del año 2016, mediante estudios de GWAS (Genome Wide Association Study), se ha descripto un nuevo SNP en el gen SLC22A3 (rs2048327) que estaría vinculado a enfermedad vascular coronaria influyendo los niveles de $\operatorname{Lp}(\mathrm{a}) .(8,9)$

En base a los nuevos conocimientos en cuanto a la base genética de la determinación de los niveles de Lp(a), utilizando modernos métodos de biología molecular, es posible estudiar tanto el número de repeticiones del KIV en el gen LPA como los SNP relacionados con mayor riesgo. (5)

La tomografía computarizada cardíaca y en especial el puntaje de calcio coronario han demostrado ser los predictores más fuertes de eventos coronarios, posibilitando la reclasificación del riesgo cardiovascular. Adicionalmente, la tomografía computarizada cardíaca ha demostrado que es altamente sensible para la evaluación de la calcificación de las estructuras relacionadas con la raíz aórtica y la aorta torácica, permitiendo establecer un diagnóstico con un método no invasivo, preciso y altamente reproducible. $(10,11)$

El objetivo principal del presente estudio es la descripción de un grupo de pacientes con elevaciones extremas de la $\mathrm{Lp}$ (a), su relación con lesiones cardíacas y vasculares evaluadas por tomografía, así como su asociación con los polimorfismos genéticos estudiados (rs10455872, rs2048327, número de repeticiones de KIV-2).

\section{MATERIAL Y MÉTODOS}

\section{Criterio de selección de los pacientes}

Se estudiaron 40 pacientes consecutivos adultos y no relacionados con niveles de $\mathrm{Lp}(\mathrm{a})>100 \mathrm{mg} / \mathrm{dl}$ medidos por técnica de inmunoturbidimetría. Los criterios de solicitud de Lp(a) fueron los siguientes: antecedente de cardiopatía isquémica precoz (mujeres $<65$ años, varones $<55$ años), historia familiar de enfermedad cardiovascular precoz y/o elevación de $\mathrm{Lp}$ (a) y finalmente enfermedad cardiovascular recurrente al margen de tratamiento con estatinas. Se excluyeron de este análisis los pacientes con diagnóstico de hipercolesterolemia familiar. Doce pacientes se encontraban en condición de prevención secundaria en el momento de su detección en base a los criterios anteriormente descriptos.

Se recolectaron todos los datos clínicos (edad, sexo, peso, talla, tensión arterial, tabaquismo), antecedentes personales y familiares para enfermedad cardiovascular y finalmente se obtuvieron los resultados de análisis bioquímicos (CT, C-LDL, C-HDL, TG, C-noHDL, urea, creatinina, filtrado glomerular, glucemia). Adicionalmente se analizó en cada caso el tratamiento recibido en el momento de incorporar al paciente al estudio.

\section{Análisis tomográfico}

Se realizó exploración tomográfica torácica con tomógrafo Siemens Emotion 16 de 16 filas de detectores. Para la adquisición de las imágenes se realizó barrido multislice obteniendo un volumen con espesor de corte de $0,675 \mathrm{~mm}$ y reconstrucciones con colimación de $3 \mathrm{~mm}$. El scan se adquirió desde ápices pulmonares hasta las cúpulas diafragmáticas con un promedio de 80 cortes por estudio, con un FOV de 40 a $44 \mathrm{~cm}$ y dosis de $110 \mathrm{kV}$ y $15 \mathrm{~mA}$. La dosis de radiación fue de 1,2 mSv. Utilizándose como criterio de calcificación un umbral de detección de 130 unidades Hounsfield, se exploró la presencia de calcificaciones en las arterias coronarias, la raíz de la aorta (anillo aórtico, trígono fibroso, unión sinotubular, velos aórticos y senos de Valsalva), la aorta torácica distal a la raíz y el anillo mitral. La presencia y extensión de las calcificaciones se evaluaron cualitativamente y se cuantificaron según el método de Agatston. (12-14) 


\section{Análisis genético molecular}

Se obtuvo ácido desoxirribonucleico $(\mathrm{ADN})$ de sangre periférica extraída por punción venosa mediante el kit comercial ADN High Pure PCR Template preparation Kit (Roche). Los SNP rs10455872 y rs2048327 se estudiaron mediante la técnica de high resolution melting (HRM). $(15,16)$ Para el caso del rs10455872 (A>G) se utilizaron los cebadores descriptos por Santos y colaboradores (7) y para el estudio del rs2048327 $(A>G)$ se emplearon cebadores diseñados específicamente para este estudio con el Software Primer Premier (PREMIER Biosoft International). Se efectuaron amplificaciones por reacción en cadena de la polimerasa (PCR) en tiempo real en el Termociclador Rotor Gene Q (Qiagen, Hilden, Alemania) utilizando una premezcla de PCR comercial (Biodynamics). Luego de las amplificaciones se efectuó un análisis de HRM. Los diferentes perfiles de melting se visualizaron utilizando los gráficos normalizados y el software de análisis de HRM Rotor Gene Q Series (Qiagen, Hilden, Alemania) para la determinación del genotipo de cada muestra.

Se midió la variación en el número de copias del Kringle IV del exón 2 del gen LPA mediante PCR en tiempo real con la metodología descripta por Kamstrup y colaboradores modificada. (6) El número de repeticiones se relativizó al gen humano de copia única beta actina y se tomó como calibrador $\mathrm{ADN}$ de pacientes con niveles normales de $\operatorname{Lp}(\mathrm{a})$, asociando un valor menor de 1 con bajo número repeticiones del Kringle IV-2.

\section{Análisis estadístico}

En cuanto a los estudios genéticos, se determinaron las frecuencias alélicas de los SNP rs10455872 y rs2048327. Se elaboró un puntaje de riesgo genético en escala de 0 a 5 , en función del número de factores de riesgo genético presentes en cada paciente. Como factores de riesgo se consideró la presencia del alelo G en los SNP rs10455872 y rs2048327 o un valor de corte $<1$ en el análisis del número de Kringle IV-2. Las variables continuas se expresaron como media \pm desviación estándar, mientras que las variables categóricas se expresaron como porcentajes.

\section{Consideraciones éticas}

Todos los pacientes firmaron un consentimiento informado para que los datos fuesen empleados con fines de estudio. Los datos se incorporaron al estudio en forma anónima con el derecho de protección de datos del paciente de acuerdo con la ley argentina $\mathrm{N}^{\circ} 25.326$.

\section{RESULTADOS}

El promedio de edad de la población estudiada fue de 53 años (62\% masculina). El valor promedio del CT fue de $225 \mathrm{mg} / \mathrm{dl}$. De los 40 pacientes evaluados, 8 (20\%) se encontraban en prevención secundaria cardiovascular.

Se consideraron calcificaciones patológicas y se tuvieron en cuenta para el análisis los siguientes criterios: toda calcificación de la raíz aórtica y aorta torácica y un valor $>100$ en el lecho coronario.

Treinta de los 40 pacientes estudiados (75\%) presentaron al menos una calcificación en algunas de las regiones estudiadas. Se detectaron 55 calcificaciones en total distribuidas de la siguiente manera: 11 pacientes presentaron una calcificación y 19 pacientes más de una calcificación (14 pacientes con dos calcificaciones, 4 pacientes con tres y un paciente presentó cuatro calcificaciones en diferentes regiones). La distribución de las calcificaciones según la región anatómica fueron:
$49 \%(\mathrm{n}=25)$ en la aorta torácica, 35\% $(\mathrm{n}=20)$ en el árbol coronario y $16 \%(\mathrm{n}=9)$ en la raíz aórtica. No se hallaron calcificaciones a nivel del anillo/válvula mitral.

Cuando se evaluó la presencia de los factores genéticos, se encontró que la frecuencia del alelo de riesgo $\mathrm{G}$ del rs10455872 fue de 0,26. En el 72\% de los pacientes estudiados el número de repeticiones del Kringle IV-2 es menor que el que presentan pacientes con niveles de $\operatorname{Lp}($ a) normales.

En cuanto al puntaje genético, en nuestra población no se encontró ningún paciente homocigota GG para el rs10455872, de modo que los valores fueron de 0 a 4; la distribución del puntaje genético fue: 0 en 7\%, 1 en $32 \%, 2$ en $28 \%, 3$ en $30 \%$ y 4 en $3 \%$.

Los 9 pacientes con calcificación en la raíz aórtica (100\%) presentaron al menos uno de los tres factores genéticos estipulados como patogénicos (puntaje $\geq 1$ ); 18 de 20 pacientes con calcificaciones del árbol coronario (90\%) y 22 de los 25 (88\%) también mostraron al menos uno de los de riesgo relacionado con los resultados moleculares.

Tres pacientes mostraron un genotipo homocigota GG para el SNP rs2048327 y presentaron calcificaciones en dos territorios diferentes (coronaria y aorta torácica).

En la Figura 1 se resumen los hallazgos genéticos vinculados a los resultados de calcificación.

En base a los resultados se efectuaron cambios en la conducta terapéutica en todos los pacientes que mostraron un puntaje genético mayor o igual a 1 y en todos los que presentaron calcificaciones tomográficas en alguno de los tres territorios evaluados.

En función del criterio establecido, 12/40 (30\%) recibían estatinas al momento de la inclusión, pasando luego de finalizar el análisis a 37/40 pacientes (92\%); 9/40 pacientes $(22 \%)$ se encontraban recibiendo aspirina, pasando a $28 / 40(70 \%)$ posteriormente (Figura 2$)$. Un paciente se encontraba con ácido nicotínico, llegando a 7 luego de la evaluación.

\section{DISCUSIÓN}

El presente estudio observacional ha relacionado elevaciones extremas de la $\mathrm{Lp}$ (a) (> $100 \mathrm{mg} / \mathrm{dl}$ ), polimorfismos genéticos relacionados con coronariopatía, patología de la raíz aórtica y la aorta torácica y tomografía axial computarizada de tórax para evaluar la calcificación de estas estructuras.

Hemos encontrado que el $75 \%$ de los pacientes presentaron calcificaciones y una clara vinculación con los factores y polimorfismos genéticos evaluados, asociados específicamente con patología cardiovascular (coronaria, valvular aórtica y aorta torácica).

Cuando se evaluó la presencia de los factores genéticos, los resultados observados fueron los siguientes: se encontró que la frecuencia del alelo de riesgo $\mathrm{G}$ del rs10455872 en nuestro grupo de estudio fue mayor que la poblacional descripta en las bases de datos internacionales $(0,26 v s .0,07)$ y no se encontraron diferencias 


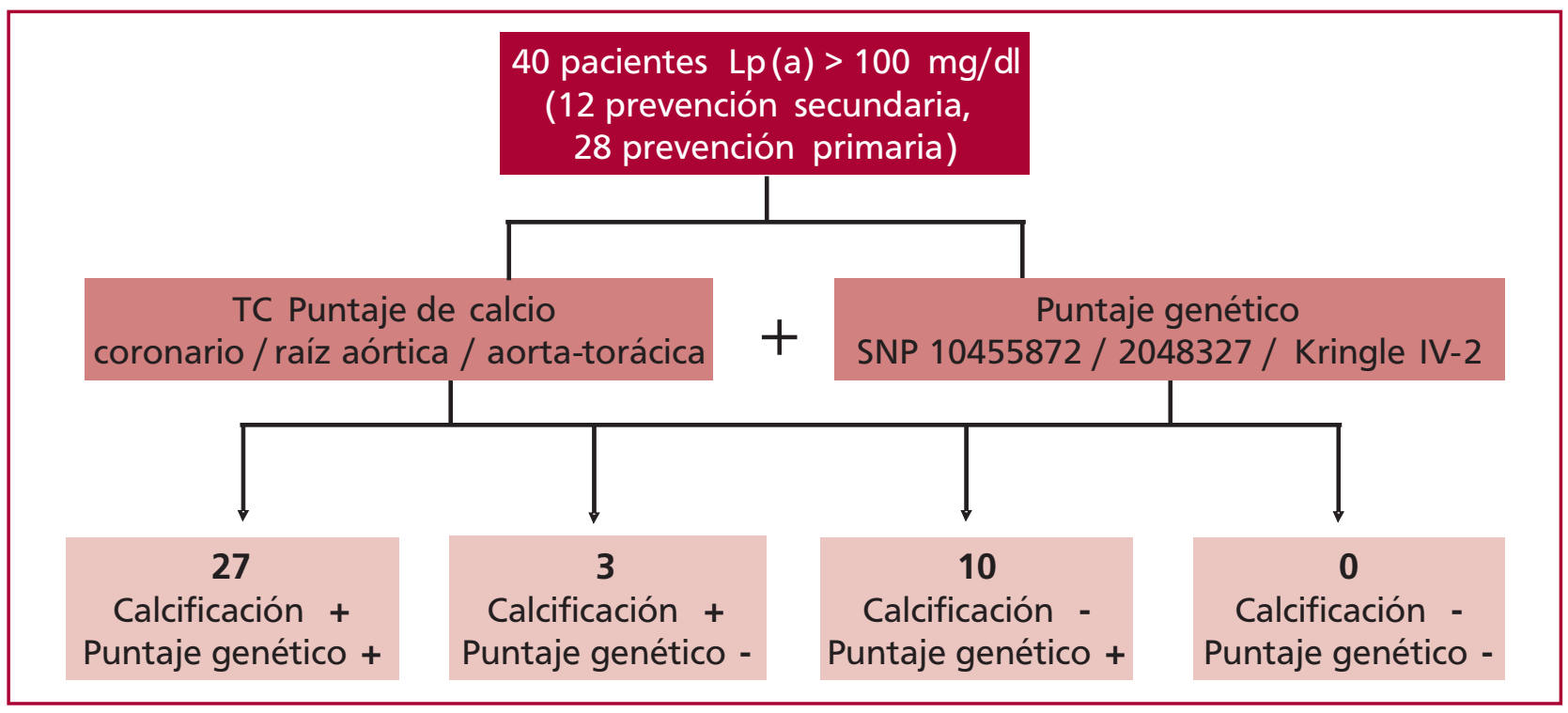

Fig. 1. Resumen de los hallazgos combinados entre calcificaciones y puntaje genético.

Fig. 2. Modificación porcentual en la utilización de estatinas y aspirina previa y posteriormente a la evaluación de cada paciente.

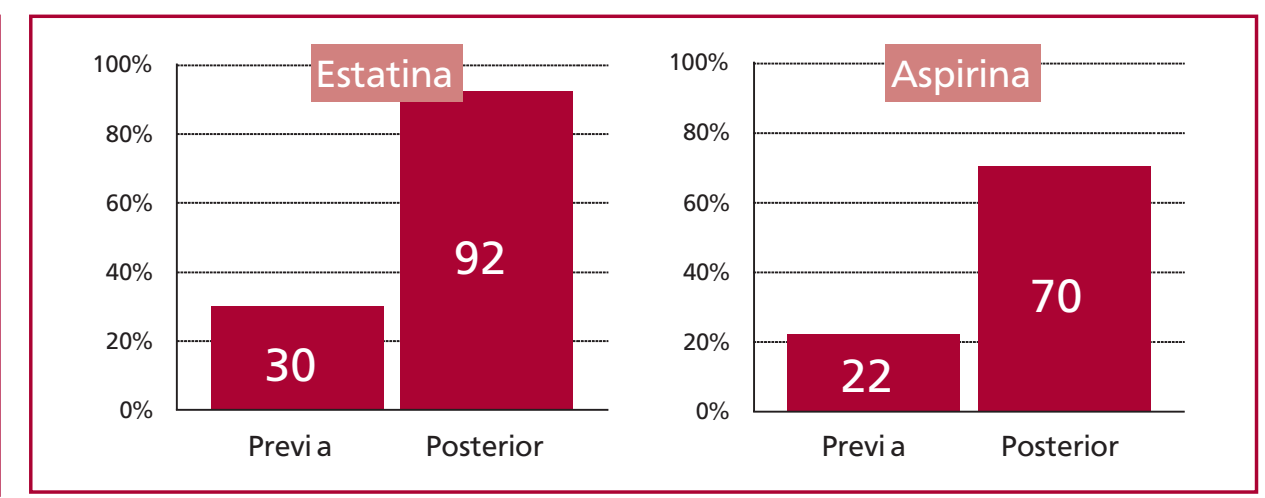

en la frecuencias alélicas de alelo $\mathrm{G}$ del polimorfismo rs2048327 (0,35 vs. 0,35). (17)

$\mathrm{La} \operatorname{Lp}(\mathrm{a})$ es una fracción lipoproteica altamente aterogénica que se encuentra regulada mayormente por factores genéticos. Las variantes genéticas, incluyendo la gran variación en el número de repeticiones del Kringle IV, le confieren a esta lipoproteína diferentes comportamientos aterogénicos. El método denominado "aleatorización mendeliana" fue aplicado hace más de dos décadas, demostrando y avalando la relación de causalidad entre este biomarcador y la ECV. (3) Este método ha posibilitado demostrar causalidad entre los niveles y genotipos específicos de $\mathrm{Lp}$ (a) y estenosis valvular aórtica. (18) El factor genético más estudiado y considerado determinante de los niveles de $\mathrm{Lp}$ (a) y sus isoformas es el Kringle denominado IV tipo 2 (KIV-2) en el gen LPA, (5) el cual hemos estudiado por primera vez en pacientes de nuestro país.

En concordancia con trabajos previos, se encontró que la frecuencia del alelo de riesgo G del rs10455872 en nuestro grupo de estudio es mayor que la poblacional $(0,26$ vs. 0,07$)$, lo cual indicaría que tiene vinculación con los niveles de Lp (a) altos que presentan estos pacientes. $(3,5,7)$ En cuanto al rs2048327, no se encontraron diferencias en la frecuencia alélica para el alelo $\mathrm{G}$ respecto de la poblacional. Si bien hubo hallazgos interesantes respecto de este SNP, como la existencia de tres pacientes homocigotas GG con calcificaciones en dos territorios diferentes (coronaria y aorta torácica), su vinculación con la regulación de los niveles $\mathrm{Lp}$ (a) recién se está iniciando, ya que fue descubierto en el corriente año (2016). (8, 9)

Acorde a lo esperado, dado que el bajo número de repeticiones se asocia con niveles altos de Lp(a), se encontró que en el $72 \%$ de los pacientes estudiados el número de repeticiones del Kringle IV-2 es menor que el que presentan pacientes con niveles de $\mathrm{Lp}$ (a) normales.

Se ha descripto que el efecto de las variantes de susceptibilidad genética es individualmente pequeño, pero sus efectos son independientes y aditivos. En un futuro, una vez que se identifiquen todas las variantes vinculadas a la determinación de los niveles de $\mathrm{Lp}(\mathrm{a})$, se podrá elaborar un puntaje de riesgo genético que consista en el número de alelos de riesgo ajustado 
por el peso de su efecto individual. (9) En el presente trabajo se utilizó un puntaje en el cual no fue posible ajustar por el peso de cada factor de riesgo dado que esta información aún se desconoce; futuros estudios permitirán contar con este tipo de herramientas que harán posible un mejor seguimiento y tratamiento de estos pacientes.

En cuanto a la asociación de los factores de riesgo genético y la calcificación, se encontró que $27 / 40$ pacientes $(67 \%)$ con al menos un marcador genético de riesgo presentaron calcificación. Diez de 40 pacientes $(25 \%)$ con puntaje genético $\geq 1$ no presentaron calcificación, lo cual estaría indicando que la presencia de estos marcadores no tiene una correlación total con la calcificación; como hemos manifestado anteriormente, algunos de estos marcadores se encuentran en evaluación en cuanto a su influencia sobre los niveles de $\operatorname{Lp}($ a) y la calcificación. Tres pacientes sin ningún factor de riesgo genético presentaron calcificación; dado que no hemos analizado todos los factores genéticos del gen LPA, no podemos descartar que estas calcificaciones se deban a la acción de otros polimorfismos relacionados con la $L p(a) u$ otros factores de riesgo cardiovascular. No encontramos ningún paciente con puntaje genético 0 que no presentara calcificaciones.

El manejo y el tratamiento aplicados a las elevaciones de la $L p(a)$ encuentran actualmente serias limitaciones; no existe una estrategia farmacológica directa para modificar los niveles de $\mathrm{Lp}$ (a). Las recomendaciones actuales establecen que ante la falta de agentes farmacológicos directos para reducir los niveles de $\operatorname{Lp}(\mathrm{a})$, la opción lógica y razonable es la de intensificar el descenso del C-LDL asociando o no la utilización de aspirina. (2)

Desde el punto de vista de la aplicabilidad clínica de nuestro estudio, nos ha permitido ajustar y adecuar las medidas farmacológicas de acuerdo con nuestros hallazgos. Así, por ejemplo, el uso de estatinas pasó de un $30 \%$ a un $92 \%$ y la utilización de aspirina, de un $22 \%$ a un $70 \%$.

Diferentes niveles y polimorfismos de $\operatorname{Lp}(\mathrm{a})$ se han descripto en distintas partes del mundo. (19) Este es el primer estudio que aporta, en parte, evidencia de la relación estrecha entre niveles, polimorfismos específicos y calcificaciones torácicas en un grupo de pacientes de nuestra región.

La relación causal entre la Lp(a) y la ECV parece clara y bien establecida en base a evidencia a diferentes niveles. Sin embargo, la falta de un recurso terapéutico eficaz, seguro, con evidencia de no solo disminuir los niveles de Lp(a), sino también de evitar eventos clínicos parece ser el último eslabón faltante en esta cadena. (20) Por lo tanto, se abre la puerta para que desde la genética y comprendiendo en detalle la fisiopatología de la relación entre la ECV y la Lp(a) se pueda avanzar en el desarrollo de diferentes estrategias para disminuir el riesgo de esta asociación.

\section{Limitaciones}

Nuestro trabajo presenta lógicas limitaciones; la falta de un grupo control debilita el análisis de causalidad. El número reducido de pacientes como limitación no debe hacer olvidar que las elevaciones extremas de la Lp(a) son sumamente infrecuentes, lo que transforma este punto en una fortaleza del estudio. $(5,6)$ En segundo lugar, desde el punto de vista del análisis de la genética molecular, debemos reconocer como limitación que no hemos estudiado todos los polimorfismos del gen LPA, que no se cuenta con datos de sus frecuencias alélicas en nuestra población y que la interpretación del número de repeticiones del Kringle IV-2 no se encuentra validada. $(4,5)$ Por último, relacionado con la tomografía axial computarizada y las calcificaciones torácicas evaluadas, si bien figura como método de elección para la detección de la aterosclerosis subclínica y calcificación valvular, podría subvalorar la presencia de placas lipídicas no calcificadas. $(11,13)$

\section{CONCLUSIÓN}

Debemos destacar que un grupo importante de pacientes con elevaciones extremas de Lp(a) presentaron calcificaciones vasculares y polimorfismos genéticos relacionados con el gen LPA. En base a estudios de genética molecular y un método no invasivo de detección de calcificación vascular y aterosclerosis subclínica hemos recategorizado y ajustado el manejo farmacológico en un número sustancial de pacientes, con el fin de disminuir su riesgo cardiovascular.

\section{Declaración de conflicto de intereses}

Los autores declaran que no poseen conflicto de intereses.

(Véanse formularios de conflicto de intereses de los autores en la web/ Material suplementario).

\section{BIBLIOGRAFÍA}

1. Berg K. A new serum type system in man. The LP system. Acta Pathol Microbiol Scand 1963;59:369-82.

2. Nordestgaard BG, Chapman MJ, Ray K, Borén J, Andreotti F, Watts GF, et al; European Atherosclerosis Society Consensus Panel. Lipoprotein(a) as a cardiovascular risk factor: current status. Eur Heart J 2010;31:2844-53. http://doi.org/fs473j

3. Clarke R, Peden JF, Hopewell JC, Kyriakou T, Goel A, Heath SC, et al; PROCARDIS Consortium. Genetic variants associated with Lp(a) lipoprotein level and coronary disease. N Engl J Med 2009;361:251828. http://doi.org/bvz7dw

4. Kamstrup PR, Tybjaerg-Hansen A, Steffensen R, Nordestgaard BG. Genetically elevated lipoprotein(a) and increased risk of myocardial infarction. JAMA 2009;301:2331-9. http://doi.org/df65xg

5. Kronenberg F. Human genetics and the causal role of lipoprotein(a) for various diseases. Cardiovasc Drugs Ther 2016;30:87-100. http:// doi.org/b2bg

6. Kamstrup PR, Tybjærg-Hansen A, Nordestgaard BG. Extreme lipoprotein(a) levels and improved cardiovascular risk prediction. J Am Coll Cardiol 2013;61:1146-56. http://doi.org/f2f3tz

7. Santos PC, Bueno CT, Lemos PA, Krieger JE, Pereira AC. LPA rs10455872 polymorphism is associated with coronary lesions in Brazilian patients submitted to coronary angiography. Lipids Health Dis $2014 ; 13: 74$. http://doi.org/b2bh 
8. Nurnberg ST, Zhang H, Hand NJ, Bauer RC, Saleheen D, Reilly MP, et al. From loci to biology: functional genomics of genome-wide association for coronary disease. Circ Res 2016;118:586-606. http:// doi.org/b2bj

9. McPherson R, Tybjaerg-Hansen A. Genetics of coronary artery disease. Circ Res 2016;118:564-78. http://doi.org/b2bk

10. Pawade TA, Newby DE, Dweck MR. Calcification in aortic stenosis: the skeleton key. J Am Coll Cardiol 2015;66:561-77. http:/ doi.org/f3jhfz

11. Hecht HS. Coronary artery calcium scanning: past, present, and future. JACC Cardiovasc Imaging 2015;8:579-96. http://doi. org $/ \mathrm{b} 2 \mathrm{bm}$

12. Agatston AS, Janowitz WR, Hildner FJ, Zusmer NR, Viamonte M Jr, Detrano R. Quantification of coronary artery calcium using ultrafast computed tomography. J Am Coll Cardiol 1990;15:827-32.

13. Alluri K, Joshi PH, Henry TS, Blumenthal RS, Nasir K, Blaha MJ. Scoring of coronary artery calcium scans: history, assumptions, current limitations, and future directions. Atherosclerosis 2015;239:10917. http://doi.org/f25vxv

14. Tota-Maharaj R, Joshi PH, Budoff MJ, Whelton S, Zeb I, Rumberger J, et al. Usefulness of regional distribution of coronary artery calcium to improve the prediction of all-cause mortality. Am J Cardiol 2015;115:1229-34. http://doi.org/f3hpnf

15. Erali M, Voelkerding KV, Wittwer CT. High resolution melting applications for clinical laboratory medicine. Exp Mol Pathol 2008;85:50-8. http://doi.org/ftc7f6

16. Vossen RH, Aten E, Roos A, den Dunnen JT. High-resolution melting analysis (HRMA): more than just sequence variant screening. Hum Mutat 2009;30:860-6. http://doi.org/ch625n

17. Base de datos de SNPs dbSNP, National Center for Biotechnology Information (http://www.ncbi.nlm.nih.gov/snp/)

18. Kamstrup PR, Tybjaerg-Hansen A, Nordestgaard BG. Elevated lipoprotein(a) and risk of aortic valve stenosis in the general population. J Am Coll Cardiol 2014;63:470-7. http://doi.org/f2qrkk

19. Khalifa M, Noureen A, Ertelthalner K, Bandegi AR, Delport R, Firdaus WJ, et al. Lack of association of rs3798220 with small apolipoprotein(a) isoforms and high lipoprotein(a) levels in East and Southeast Asians. Atherosclerosis 2015;242:521-8. http://doi.org/f3jhw2 20. Tsimikas S, Viney NJ, Hughes SG, Singleton W, Graham MJ, Baker BF, et al. Antisense therapy targeting apolipoprotein(a): a randomised, double-blind, placebo-controlled phase 1 study. Lancet 2015;386:1472-83. http://doi.org/f3jh94 